\title{
ON SOME MATHEMATICAL MODELS OF FILTRATION THEORY
}

\author{
S.G. Pyatkov, Ugra State University, Khanty-Mansyisk, Russian Federation, \\ S_pyatkov@ugrasu.ru, \\ S.N. Shergin, Ugra State University, Khanty-Mansyisk, Russian Federation, Ssn@ugrasu.ru
}

The article is devoted to the study of some mathematical models arising in filtration theory. We examine an inverse problem of determining an unknown right-hand side and coefficients in a pseudoparabolic equation of the third order. Equations of this type and more general Sobolev-type equations arise in filtration theory, heat and mass transfer, plasma physics, and in many other fields. We reduce the problem to an operator equation whose solvability is established with the help of a priori estimates and the fixed point theorem. Together with the natural smoothness conditions for the data, we require also some well-posedness condition to be fulfilled which is actually reduced to the condition of nondegeneracy of some matrix constructed with the use of the data of the problem. Theorems on existence and uniqueness of solutions to this problem are stated and proven. Stability estimates are exposed. In the linear case the result is global in time, while in the nonlinear case it is local. The main function spaces used are the Sobolev spaces.

Keywords: pseudoparabolic equation; existence and uniqueness theorem; inverse problem; boundary value problem.

\section{Introduction}

Together with a solution $U$, we determine an unknown right-hand side and coefficients of the equation

$$
L U_{t}+M U=f, \quad(x, t) \in Q=G \times(0, T),
$$

where $L, M$ are the second-order differential operators in the variables $x$ and $G$ is a bounded domain in $R^{n}(n \geq 1)$ with boundary $\Gamma \in C^{2}$. The equation is complemented with the initial and boundary conditions

$$
\begin{gathered}
\left.U\right|_{S}=\varphi, \quad S=\Gamma \times(0, T), \\
\left.U\right|_{t=0}=U_{0}(x) .
\end{gathered}
$$

We employ the values of a solution $U$ at separate points as overdetermination conditions. So, our overdetermination conditions are as follows:

$$
U\left(x_{i}, t\right)=\alpha_{i}(t), \quad(i=1,2, . ., r)
$$

with $x_{i}$ being arbitrary points in $G$.

Mathematical models based on the pseudoparabolic equations arise when describing heat and mass transfer, filtration, wave processes, and many other processes [1, 2]. Many articles are devoted to the study of solvability of boundary value problems for pseudoparabolic equations (see, for instance, [3, 4]). In particular, initial, initial-boundary, and periodic problems, the questions of global (in time) solvability and blow-up of solutions are studied. In the case of global solvability some investigations are devoted to the questions of asymptotic behavior of solutions to the direct problems, scattering theory, and stability of soliton-type solutions to both 
one-dimensional and multidimensional equations, in particular, to the Benjamin-Bona-MahonyBurgers and Rosenau-Burgers equations [5,6]. The semigroup approach to singular Sobolevtype equations was developed by Sviridyuk and Fedorov [4]. Some results devoted to the theory of pseudoparabolic equations with an indefinite or noninvertible operator at the higher-order derivative with respect to time can be found in [7]. The questions of local solvability for nonlinear pseudoparabolic equations are examined in [8].

The pseudoparabolic equations with a monotone nonlinearity are studied by R.E. Showalter in [9], where the classical monotonicity method in an expanded form was applied to various classes of equations of mathematical physics, in particular, to nonlinear Sobolev-type equations with monotone nonlinearities. The existence of global (in time) solutions for the Boussinesq equation with a source and finite-time blow-up solutions were studied by Kozhanov in [10]. In his articles the blow-up of solutions to the first boundary value problem is proven with the use of the comparison principle for these equations. In particular, the blow-up of a positive solution is proven and some existence and nonexistence theorems are exhibited.

The question of uniqueness of solutions to the Cauchy problem for the quasilinear pseudoparabolic equation

$$
u_{t}=c \triangle u_{t}+\varphi(u)
$$

is studied in [11] in a class of growing functions $\varphi(u)$, with $u$ from some well-posedness class. The maximum principle for pseudoparabolic equations is also presented in [12]. The method of the proof of nonexistence of solutions to some boundary value problems relying on the maximum principle is developed in the articles by Yu.V. Egorov and V.A. Kondratev. The methods of complex analysis are employed in the study of pseudoparabolic equations in [13]. A principally new approach called the method of test functions was proposed in [14, 15]. The existence and nonexistence questions for different mathematical models on the base of Sobolev-type equations are presented in the well-known monograph [2], where the necessary bibliography can be found.

The inverse problems for Sobolev-type equations are not studied well. In $[1,16]$ the authors consider a model that discribes filtration of a fluid in a fractured media. They state some inverse problems, including a problem close to that of out article, and establish local existence and uniqueness theorems under an integral overdetermination condition on the boundary. Moreover, some properties of solutions to inverse problems of this type are derived there. The problem of recovering of a kernel of an integral operator occurring in Sobolev-type equations when some functional of a solution is given is examined in [17]. The coefficient inverse problems are considered in [18]. The uniqueness theorem is proven and an algorithm for solving an inverse problem for the equation

$$
u_{t}-\Delta u_{t}=a \Delta u+b(y) u_{y}+c(y)+\delta(t, x, y), \quad(x, y) \in R^{2}, t>0,
$$

is specified. The unknowns are $u(t, x), b(y), c(y)$, and a constant $a$ and the functions $u(t, x, 0), u_{y}(t, x, 0)$, and $u(0, x, y)$ are given. Here $\delta(t, x, y)$ is the Dirac delta function.

One more problem is considered in [19], where the value of a functional of a solution allows us to recover a scalar function depending on $t$ which is a factor before an element of a given Banach space on the right-hand side of an abstract Sobolev-type equation. A similar problem is treated in [20], where an element of a Banach space is recovered under the integral overdetermination condition. We can refer also to [21], where some inverse problems for composite type equations are considered.

Point out a series of monographs and articles [22-26], where essential advancements in the theory of inverse problems for parabolic equations and systems are made. In particular, the inverse problems in the same statement but for parabolic equations and systems are treated in [27-29].

The main result of the article is Theorem 3 , where existence and uniqueness of a solution to the problem (1) - (4) are proven and a stability estimate is obtained. The problem of determination 
a right-hand side (a source function) is linear and the result in this case is global in time (see [30]).

\section{Preliminaries}

In the article we employ the Sobolev spaces $W_{p}^{s}(G)$ and the Hölder spaces $C^{\alpha}(\bar{G})$. The space of strongly measurable functions on $[0, T]$ with values in a Banach space $H$ is denoted by $L_{p}(0, T ; H)$. The condition $\Gamma \in C^{2 m}$ means that, for every $x_{0} \in \Gamma$, there exists a neighborhood $U$ (the coordinate neighborhood) and a coordinate system $y$ (local coordinate system), obtained after a rotation and translation of the initial system in which

$$
\begin{gathered}
\bar{U} \cap G=\left\{y \in \mathbb{R}^{n}: y^{\prime} \in \overline{B_{r}}, \omega\left(y^{\prime}\right)<y_{n} \leq \omega\left(y^{\prime}\right)+\delta\right\}, \\
\bar{U} \cap\left(\mathbb{R}^{n} \backslash \bar{G}\right)=\left\{y \in \mathbb{R}^{n}: \omega\left(y^{\prime}\right)-\delta \leq y_{n}<\omega\left(y^{\prime}\right)\right\}, \\
\Gamma \cap \bar{U}=\left\{y \in \mathbb{R}^{n}: y^{\prime} \in \overline{B_{r}}, y_{n}=\omega\left(y^{\prime}\right)\right\},
\end{gathered}
$$

where $y^{\prime}=\left(y_{1}, y_{2}, \ldots, y_{n-1}\right), B_{r}=\left\{y^{\prime}:\left|y^{\prime}\right|<r\right\}, \delta>0$ is a constant, and $\omega \in C^{2 m}\left(\overline{B_{r}}\right)$. Without loss of generality, we can assume that the axis $y_{n}$ of the local coordinate system is directed along the normal to $\Gamma$ at $x_{0}$.

Let $L$ and $M$ be second-order operators of the form:

$$
\begin{aligned}
& L U=\sum_{i, j=1}^{m} a_{i j}(x, t) U_{x_{i} x_{j}}+\sum_{i=1}^{m} a_{i}(x, t) U_{x_{i}}+a_{0}(x, t) U, \\
& M U=\sum_{i, j=1}^{m} b_{i j}(x, t) U_{x_{i} x_{j}}+\sum_{i=1}^{m} b_{i}(x, t) U_{x_{i}}+b_{0}(x, t) U .
\end{aligned}
$$

Here $L$ is assumed to be elliptic. Thus, there exists a constant $\delta>0$ such that

$$
\sum_{i, j=1}^{m} a_{i j} \xi_{i} \xi_{j} \geq \delta_{0}|\xi|^{2} \quad \forall \xi \in R^{n}, \quad \forall(x, t) \in \bar{Q} .
$$

Write out the conditions on the coefficients of $L, M$. Fix a parameter $p>n$ and assume that

$$
\begin{gathered}
a_{i j} \in C(\bar{Q}), \quad a_{i}, a_{0} \in C\left([0, T] ; L_{p}(G)\right) \quad(i, j=1,2, . ., n), \\
a_{0}(x, t) \leq 0 \text { a.e. in } Q, \\
b_{i j} \in L_{p}\left(0, T ; L_{\infty}(G)\right), b_{i}, b_{0} \in L_{p}(Q)(i, j=1,2, . ., n) .
\end{gathered}
$$

Under these conditions on the coefficients of $L$, the following theorem is valid.

Theorem 1. For every $f \in L_{p}(Q)$, the Dirichlet problem

$$
L u=f,\left.\quad u\right|_{\Gamma}=0,
$$

has a unique solution $u \in L_{p}\left(0, T ; W_{p}^{2}(G)\right)$ satisfying the estimate

$$
\|u\|_{W_{p}^{2}(G)} \leq c\|f\|_{L_{p}(G)} \text { almost everywhere on }(0, T),
$$

where $c$ is a constant independent of $f$ and $t$.

Proof. The solvability of problems (8) (depending on a parameter $t$ ) results from the uniqueness of solutions (see the maximum principle in [31, Chap. 9]) and the Fredholm property of these 
problems. An estimate for solutions can be justified with the use of the continuity of coefficients in $t$; indeed, in some neighborhood of every point $t_{0} \in[0, T]$ there is an estimate of the form $\|u\|_{W_{p}^{2}(G)} \leq c\|L u\|_{L_{p}(G)}$ with a constant $c$ independent of $t$. These estimate ensure the presence of a global estimate which is claimed.

Lemma 1. If $b \in L_{q}(G)$ with $q>n$ for $p \leq n$ and $q \geq p$ for $p>n$ then there exists a constant $c>0$ such that for all functions $u \in W_{p}^{2}(G)$ the following estimate is valid:

$$
\|b \nabla U\|_{L_{p}(G)} \leq c\|b\|_{L_{q}(G)}\|U\|_{W_{p}^{2}(G)} .
$$

If $b \in L_{q}(G)$ with $q>n / 2$ for $p \leq n / 2$ and $q \geq p$ for $p>n / 2$ then there exists a constant $c>0$ such that, for all functions $u \in W_{p}^{2}(G)$, we have

$$
\|b U\|_{L_{p}(G)} \leq c\|b\|_{L_{q}(G)}\|U\|_{W_{p}^{2}(G)} .
$$

Proof. Prove the former statement, the latter is established by analogy. Fix $i=1,2, \ldots, n$. The Hölder inequality yields

$$
\left\|b U_{x_{i}}\right\|_{L_{p}(G)}=\left(\int_{G}|b|^{p}\left|U_{x_{i}}\right|^{p} d x\right)^{1 / p} \leq\left(\int_{G}|b|^{q} d x\right)^{\frac{1}{q}}\left(\int_{G}\left|U_{x_{i}}\right|^{\frac{p q}{q-p}} d x\right)^{\frac{q-p}{q p}} .
$$

By the embedding theorems (see [34]), we have the estimate

$$
\left\|U_{x_{i}}\right\|_{L_{\frac{q p}{q-p}}(G)} \leq c\|U\|_{W_{p}^{2}(G)}, \quad q>n, p \leq n .
$$

Thus

$$
\left\|b U_{x_{i}}\right\|_{L_{p}(G)} \leq c\|U\|_{W_{p}^{2}(G)}, \quad c=\|b\|_{L_{q}(G)} .
$$

The estimate (10) is obtained similarly.

Let $Q^{\gamma}=G \times(0, \gamma)$.

Theorem 2. (solvability of the direct problem). Assume that $f \in L_{p}(Q), U_{0}(x) \in W_{p}^{2}(G)$ and $\varphi_{t} \in L_{p}\left(0, T ; W_{p}^{2-1 / p}(G)\right)$, where $p \in(n, \infty)$, and conditions (6), (7) for the coefficients hold together with the consistency conditions

$$
\left.\varphi(x, 0)\right|_{\Gamma}=\left.U_{0}(x)\right|_{\Gamma}
$$

Then there exists a unique solution to problem (1) - (3) such that

$$
U, U_{t} \in L_{p}\left(0, T ; W_{p}^{2}(G)\right), \quad U(t) \in C\left([0, T] ; W_{p}^{2}(G)\right) .
$$

If $\varphi \equiv 0, \quad U_{0}(x) \equiv 0$, then there exists a constant $c>0$ independent of $\gamma \in[0, T]$ such that a solution to problem (1) - (3) meets the estimate

$$
\|U\|_{L_{\infty}\left(0, \gamma ; W_{p}^{2}(G)\right)}+\left\|U_{t}\right\|_{L_{p}\left(0, \gamma ; W_{p}^{2}(G)\right)} \leq c\|f\|_{L_{p}\left(Q^{\gamma}\right)}
$$

Proof. Consider the segment $[0, T]$. Find a function $\Phi$ such that

$$
\Phi_{t} \in L_{p}\left(0, T ; W_{p}^{2}(G)\right):\left.\quad \Phi\right|_{S}=\varphi,\left.\quad \Phi\right|_{t=0}=U_{0}(x) .
$$


It can be constructed as follows:

$$
\Phi=\psi+U_{0}, \quad \psi=\int_{0}^{t} \psi_{0}(x, \tau) d \tau, \quad \Delta \psi_{0}=0,\left.\quad \psi_{0}\right|_{\Gamma}=\varphi_{t}
$$

(the existence of a function $\psi_{0}$ follows from the known results (see, for instance, [31]). Make the change $V=U-\Phi$. Function $V$ satisfies the conditions

$$
L V_{t}+M V=f-L \Phi_{t}-M \Phi=g,\left.\quad V\right|_{S}=0,\left.\quad V\right|_{t=0}=0 .
$$

Using Theorem 1 we can obtain that

$$
V+\int_{0}^{t} L^{-1} M V(\tau, x) d \tau=L^{-1} g
$$

Conditions (6) and Lemma 1 justify the estimate $(\tau \leq T)$

$$
\left\|\int_{0}^{t} L^{-1} M V d \tau\right\|_{C\left([0, \tau] ; W_{p}^{2}(G)\right)} \leq c \tau^{1 / q}\|V\|_{C\left([0, \tau] ; W_{p}^{2}(G)\right)},
$$

where $1 / p+1 / q=1$, which together with the fixed point theorem allows us to prove the claim on solvability on some time interval $\left[0, \tau_{0}\right]\left(c \tau_{0}^{1 / q}<1\right)$. The global theorem results by repetition of the arguments at the segment $\left[\tau_{0}, 2 \tau_{0}\right],\left[2 \tau_{0}, 3 \tau_{0}\right]$, etc. It is easy to see that a solution meets the estimate from the theorem.

Consider the interval $(0, \gamma)(\gamma \leq T)$ and prove the estimate of the theorem. Consider problem $(1)-(3),(4)$, where $U_{0}=0, \varphi=0$, and

$$
f_{0}=\left\{\begin{array}{ll}
f, & t \leq \gamma \\
0, & t>\gamma
\end{array} \quad \in L_{p}(Q)\right.
$$

There exists a unique solution $\tilde{U}$ to problem (1) - (3) satisfying the estimate:

$$
\|\tilde{U}\|_{L_{\infty}\left(0, T ; W_{p}^{2}(G)\right)}+\left\|\tilde{U}_{t}\right\|_{L_{p}\left(0, T ; W_{p}^{2}(G)\right)} \leq c\left\|f_{0}\right\|_{L_{p}(Q)}=c\|f\|_{L_{p}\left(Q^{\gamma}\right)} .
$$

By the uniqueness theorem, a solution $\tilde{U}$ agrees with a solution $U$ to the problem (1) - (3), with $U_{0}=0$ and $\varphi=0$ on $[0, \gamma]$. Thus, we can rewrite (13) as follows

$$
\|U\|_{L_{\infty}\left(0, \gamma ; W_{p}^{2}(G)\right)}+\left\|U_{t}\right\|_{L_{p}\left(0, \gamma ; W_{p}^{2}(G)\right)} \leq c\|f\|_{L_{p}\left(Q^{\gamma}\right)} .
$$

Remark. We can easily formulate an analogue of this theorem for any $p \in(1, \infty)$. But to obtain the main results we need a condition $p>n$ for $p$. Hence, we state the above theorems in this case.

\section{The Main Results}

We consider the inverse problem of determining the unknown functions occurring in the righthand side of the equation, and in the operator itself. In this case the problem is nonlinear. We assume that the right-hand side of (1) is represented as

$$
f=\sum_{i=1}^{r_{0}} c_{i}(t) f_{i}(x, t)+f_{0}(x, t), \quad f_{i} \in L_{\infty}\left(0, T ; L_{p}(G)\right) \quad\left(i=1,2, . ., r_{0}\right),
$$


where the functions $f_{i}$ are given. We suppose also that some coefficients of the operator $M$ depending on $t$ are unknown and operator $M$ has the form

$$
\begin{gathered}
M U=M_{r_{0}} U+\sum_{k=r_{0}+1}^{r} c_{k}(t) M_{k} U \\
M_{k} U=\sum_{i, j=1}^{n} b_{i j}^{k}(x, t) U_{x_{i} x_{j}}+\sum_{i=1}^{n} b_{i}^{k}(x, t) U_{x_{i}}+b_{0}^{k}(x, t) U .
\end{gathered}
$$

\section{Consistency conditions:}

$$
\alpha_{i}(0)=U_{0}\left(x_{i}, t\right), \quad(i=1,2, . ., r) .
$$

Construct the function $\Phi \in C\left([0, T] ; W_{p}^{2}(G)\right)(p>n)$ such that $\Phi_{t} \in L_{p}\left(0, T ; W_{p}^{2}(G)\right)$, $\left.\Phi\right|_{t=0}=U_{0}(x),\left.\Phi\right|_{S}=\varphi$ (see the proof of theorem 2). Construct also the matrix $B$ with the rows

$$
L^{-1} f_{1}\left(x_{j}, t\right), L^{-1} f_{2}\left(x_{j}, t\right), \ldots, L^{-1} f_{r_{0}}\left(x_{j}, t\right),-L^{-1} M_{r_{0}+1} \Phi\left(x_{j}, t\right), \ldots,-L^{-1} M_{r} \Phi\left(x_{j}, t\right),
$$

where $j=1,2, \ldots, r$, and assume that there exists $\delta_{0}>0$ such that

$$
|\operatorname{det} B| \geq \delta_{0} \text { a.e. on }[0, T] \text {. }
$$

Here $L^{-1} f_{i}$ is a solution $U_{i}$ to the problem $L U_{i}=f_{i},\left.U_{i}\right|_{t=0}=0,\left.U_{i}\right|_{S}=0$. We assume that the coefficients of the operator

$$
L U=\sum_{i, j=1}^{n} a_{i j}(x, t) U_{x_{i} x_{j}}+\sum_{i=1}^{n} a_{i}(x, t) U_{x_{i}}+a_{0}(x, t) U,
$$

meet conditions (6) and the coefficients of $M_{i}$ the conditions

$$
b_{i j}^{k} \in L_{\infty}(Q), b_{i}^{k}, b_{0}^{k} \in L_{\infty}\left(0, T ; L_{p}(G)\right) \quad\left(i, j=1,2, . ., n, k=r_{0}, \ldots, r\right) .
$$

In this case, locally in time, conditions (16) does not depend on the choice of function $\Phi$. Indeed, let $\Phi_{1}$ and $\Phi_{2}$ be such functions. Consider

$$
\begin{gathered}
\left|L^{-1} M_{i} \Phi_{1}\left(x_{j}, t\right)-L^{-1} M_{i} \Phi_{2}\left(x_{j}, t\right)\right| \leq c\left\|M_{i} \Phi_{1}(x, t)-M_{i} \Phi_{2}(x, t)\right\|_{L_{p}(G)} \leq \\
\leq c\left\|\Phi_{1}(x, t)-\Phi_{2}(x, t)\right\|_{W_{p}^{2}(G)} \leq c t^{1 / q}\left\|\Phi_{1 t}-\Phi_{2 t}\right\|_{L_{p}\left(0, T ; W_{p}^{2}(G)\right)} .
\end{gathered}
$$

This inequality shows that if $B_{1}$ and $B_{2}$ are matrices constructed using $\Phi_{1}$ and $\Phi_{2}$, respectively, and $\left|\operatorname{det} B_{1}\right| \geq \delta_{0}>0$, then we can find $\gamma_{0}>0$ such that, for $t \in\left[0, \gamma_{0}\right],\left|\operatorname{det} B_{2}\right| \geq \delta_{0} / 2>0$ and thus on the interval $\left[0, \gamma_{0}\right]\left|\operatorname{det} B_{i}\right| \geq \delta_{0} / 2>0$ for $i=1,2$.

Theorem 3. Let conditions (5), (6), (15) - (17) be fulfilled. Then there exists a constant $\gamma_{0}>0$ such that on the interval $\left[0, \gamma_{0}\right]$ problem $(1)-(4)$ has a unique solution $\left(U, c_{1}, \ldots, c_{r}\right)$ such that

$$
U \in C\left(\left[0, \gamma_{0}\right] ; W_{p}^{2}(G)\right), \quad U_{t} \in L_{p}\left(\left[0, \gamma_{0}\right] ; W_{p}^{2}(G)\right), \quad c_{i}(t) \in L_{p}\left(0, \gamma_{0}\right)(i=1,2, \ldots, r),
$$

Proof. Let

$$
\Phi \in C\left([0, T] ; W_{p}^{2}(G)\right): \quad \Phi_{t} \in L_{p}\left([0, T] ; W_{p}^{2}(G)\right)
$$

be a solution to the problem (see Theorem 2)

$$
L \Phi_{t}+M_{r_{0}} \Phi=f_{0},\left.\quad \Phi\right|_{t=0}=U_{0},\left.\quad \Phi\right|_{S}=\varphi .
$$


In this case the function $V=U-\Phi$ is a solution to the problem

$$
L V_{t}+M V=\sum_{i=1}^{r_{0}} c_{i}(t) f_{i}(x, t)-\sum_{i=r_{0}+1}^{r} c_{i}(t) M_{i} \Phi,\left.\quad V\right|_{t=0}=0,\left.\quad V\right|_{S}=0
$$

There exists $\gamma_{0}>0$ such that condition (16), with $\delta_{0} / 2$ rather than $\delta_{0}$, holds on $\left[0, \gamma_{0}\right]$. Note that $U$ is solution to problem (1) - (4). In this case

$$
V\left(x_{j}, t\right)=\alpha_{j}(t)-\Phi\left(x_{j}, t\right)=\tilde{\alpha}_{j}(t) .
$$

The function $\Phi \in W_{p}^{2}\left(G ; W_{p}^{1}(0, T)\right)$ after a possible modification on a set of zero measure possesses the property $\Phi \in C^{\alpha}\left(\bar{G} ; W_{p}^{1}(0, T)\right), \alpha \leq 2-\frac{n}{p}$ (see (5.4) in [35]). In particular, $\Phi\left(x_{i}, t\right) \in W_{p}^{1}(0, T)$, and thus

$$
\tilde{\alpha}_{j}(t) \in W_{p}^{1}(0, T) \quad(j=1,2, . ., r) .
$$

Inverting $L$ in (18), we have

$$
V_{t}+L^{-1} M V=\sum_{i=1}^{r_{0}} c_{i}(t) L^{-1} f_{i}(x, t)-\sum_{i=r_{0}+1}^{r} c_{i}(t) L^{-1} M_{i} \Phi .
$$

Note that $L^{-1} M_{i} \Phi\left(x_{j}, t\right)$ and $L^{-1} M_{i} \Phi\left(x_{j}, t\right) \in L_{\infty}(0, T)$ for almost all $t$. Actually, $L^{-1} M_{i} \Phi(x, t) \in W_{p}^{2}\left(G ; L_{p}(0, T)\right)$ and thereby $L^{-1} M_{i} \Phi\left(x_{j}, t\right) \in L_{p}(0, T)$ (see [35, (5.4)]). In this case we have

$$
\begin{gathered}
\left\|L^{-1} M_{i} \Phi\left(x_{j}, t\right)\right\|_{L_{\infty}(0, T)} \leq\left\|M_{i} \Phi\left(x_{j}, t\right)\right\|_{L_{\infty}\left(0, T ; L_{p}(G)\right)} \leq \\
c\|\Phi(x, t)\|_{L_{\infty}\left(0, T ; W_{p}^{2}(G)\right)} \leq c\|\Phi\|_{L_{\infty}\left(0, T ; W_{p}^{2}(G)\right)}
\end{gathered}
$$

Thus,

$$
\left\|L^{-1} M_{i} \Phi\left(x_{j}, t\right)\right\|_{L_{\infty}(0, T)} \leq c\|\Phi\|_{C\left([0, T] ; W_{p}^{2}(G)\right)} .
$$

Similarly, we can prove that $L^{-1} f_{i}\left(x_{j}, t\right) \in L_{\infty}(0, T)$ and this trace is defined. Let $x=x_{j}$ in $(21)$. We have

$$
V_{t}\left(x_{j}, t\right)+L^{-1} M V\left(x_{j}, t\right)=\sum_{i=1}^{r_{0}} c_{i}(t) L^{-1} f_{i}\left(x_{j}, t\right)-\sum_{i=r_{0}+1}^{r} c_{i}(t) L^{-1} M_{i} \Phi\left(x_{j}, t\right) .
$$

The overdetermination condition yields

$$
\begin{gathered}
\tilde{\alpha}_{j t}+L^{-1} M_{r_{0}} V\left(x_{j}, t\right)+\sum_{i=r_{0}+1}^{r} c_{i}(t) L^{-1} M_{i} V\left(x_{j}, t\right)=\sum_{i=1}^{r_{0}} c_{i}(t) L^{-1} f_{i}\left(x_{j}, t\right)- \\
-\sum_{i=r_{0}+1}^{r} c_{i}(t) L^{-1} M_{i} \Phi\left(x_{j}, t\right) \quad(j=1,2, . ., r) .
\end{gathered}
$$

We can rewrite equation (23) in the form

$$
B \vec{c}=\left(\begin{array}{c}
\tilde{\alpha}_{1 t}+L^{-1} M_{r_{0}} V\left(x_{1}, t\right)+\sum_{i=r_{0}+1}^{r} c_{i}(t) L^{-1} M_{i} V\left(x_{1}, t\right) \\
\ldots \\
\ldots \\
\tilde{\alpha}_{r t}+L^{-1} M_{r_{0}} V\left(x_{r}, t\right)+\sum_{i=r_{0}+1}^{r} c_{i}(t) L^{-1} M_{i} V\left(x_{r}, t\right)
\end{array}\right) .
$$


As a result, we infer

$$
\vec{c}=B^{-1}\left(\begin{array}{c}
\tilde{\alpha}_{1 t}+L^{-1} M_{r_{0}} V\left(x_{1}, t\right)+\sum_{i=r_{0}+1}^{r} c_{i}(t) L^{-1} M_{i} V\left(x_{1}, t\right) \\
\cdots \\
\ldots \\
\tilde{\alpha}_{r t}+L^{-1} M_{r_{0}} V\left(x_{r}, t\right)+\sum_{i=r_{0}+1}^{r} c_{i}(t) L^{-1} M_{i} V\left(x_{r}, t\right)
\end{array}\right)=R(\vec{c}) .
$$

The right-hand side can be viewed as an operator $R(\vec{c})$ taking the vector $\vec{c}(t)$ in a solution $V(x, t)$ to problem (18) and then taking $V(x, t)$ into the right-hand side of (25). Investigate properties of this operator. Fix

$$
R_{0}=2\left\|B^{-1} \overrightarrow{\tilde{\alpha}}\right\|_{L_{p}(0, T)}, \quad \overrightarrow{\tilde{\alpha}}=\left(\begin{array}{c}
\tilde{\alpha}_{1 t} \\
. . \\
\tilde{\alpha}_{r t}
\end{array}\right) .
$$

By construction, $\tilde{\alpha}_{i t} \in L_{p}(0, T)$. Due to the fact that the entries of the matrix $B$ belong to $L_{\infty}(0, T)$, we have that $B^{-1} \overrightarrow{\tilde{\alpha}} \in L_{p}(0, T)$. By Theorem 2 , for every vector-function

$$
\vec{c}(t) \in B_{R_{0}, \gamma}=\left\{\vec{c} \in L_{p}(0, \gamma):\|\vec{c}\|_{L_{p}(0, \gamma)} \leq R_{0}\right\}, \quad \gamma \leq T,
$$

the problem

$$
L V_{t}+M V=\sum_{i=1}^{r_{0}} c_{i}(t) f_{i}(x, t)-\sum_{i=r_{0}+1}^{r} c_{i}(t) L^{-1} M_{i} \Phi,\left.\quad V\right|_{t=0}=0,\left.\quad V\right|_{S}=0
$$

is uniquely solvable. For $\lambda>0$, we have that

$$
\sup _{t} e^{-\lambda t}\|V(t, x)\|_{W_{p}^{2}(G)} \leq \frac{1}{|\lambda|^{1 / q}}\left(\int_{0}^{\gamma}\left\|V_{t}\right\|_{W_{p}^{2}(G)}^{p} e^{-\lambda t p} d t\right)^{1 / p}
$$

From (26) it follows that

$$
\begin{gathered}
\|\| V_{t}\left\|_{W_{p}^{2}(G)} e^{-\lambda t}\right\|_{L_{p}(0, \gamma)} \leq c\left(\|\| M V\left\|_{W_{p}^{2}(G)} e^{-\lambda t}\right\|_{L_{p}(0, \gamma)}+\right. \\
\left.\|\| \sum_{i=1}^{r_{0}} c_{i}(t) f_{i}(x, t)-\sum_{i=r_{0}+1}^{r} c_{i}(t) L^{-1} M_{i} \Phi\left\|_{L_{p}(G)} e^{-\lambda t}\right\|_{L_{p}(0, \gamma)}\right) \leq \\
\left.\leq \frac{c_{0}}{|\lambda|^{1 / q}}\left(\|\vec{c}\|_{L_{p}(0, \gamma)}+1\right)\left\|V_{t} e^{-\lambda t}\right\|_{L_{p}\left(0, \gamma ; W_{p}^{2}(G)\right)}\right)+c_{1}\|\vec{c}\|_{L_{p}(0, \gamma)},
\end{gathered}
$$

where constant $c_{0}$ is independent of $\lambda>0, \gamma \leq T$. Choosing a sufficiently large $\lambda>0$ and estimating $\|\vec{c}\|_{L_{p}(0, \gamma)}$ through $R_{0}$ we obtain

$$
\left\|V_{t}\right\|_{L_{p}\left(0, \gamma ; W_{p}^{2}(G)\right)}+\|V\|_{L_{p}\left(0, \gamma ; W_{p}^{2}(G)\right)} \leq c_{2}\|\vec{c}\|_{L_{p}(0, \gamma)} \leq c\left(R_{0}\right)
$$

where $c\left(R_{0}\right)$ is independent of $\vec{c} \in B_{R_{0}, \gamma}$. Obtain the remaining estimates. Let $\vec{c}^{1}, \vec{c}^{2}$ be a vectorfunction from $L_{p}(0, \gamma)$ and $V^{1}, V^{2}$ be corresponding solutions to (26). Thus, we infer

$$
L V_{t}^{i}+M_{r_{0}} V^{i}+\sum_{j=r_{0}+1}^{r} c_{j}^{i}(t) M_{j} V^{i}=\sum_{j=1}^{r_{0}} c_{j}^{i}(t) f_{j}(x, t)-\sum_{j=r_{0}+1}^{r} c_{j}^{i}(t) M_{j} \Phi .
$$


Note that the functions $V^{i}$ satisfy estimate (29). Subtracting equations (30) for $i=1,2$ and denoting $\omega=V^{1}-V^{2}$, we arrive at the relation

$$
\begin{gathered}
L \omega_{t}+M_{r_{0}} \omega+\sum_{j=r_{0}+1}^{r} c_{j}^{1}(t)\left(M_{j} \omega\right)=\sum_{j=1}^{r_{0}}\left(c_{j}^{1}-c_{j}^{2}\right)(t) f_{i}(x, t)- \\
-\sum_{j=r_{0}+1}^{r}\left(c_{j}^{1}-c_{j}^{2}\right)(t) M_{j} V^{2}-\sum_{j=r_{0}+1}^{r}\left(c_{j}^{1}-c_{j}^{2}\right)(t) M_{j} \Phi .
\end{gathered}
$$

Inequality (29) and the previous arguments yield

$$
\left\|\omega_{t}\right\|_{L_{p}\left(0, \gamma ; W_{p}^{2}(G)\right)}+\|\omega\|_{L_{p}\left(0, \gamma ; W_{p}^{2}(G)\right)} \leq c_{2}\left\|\overrightarrow{c^{1}}-\overrightarrow{c^{2}}\right\|_{L_{p}(0, \gamma)}
$$

Also we have estimate (29), where the constant $c_{3}$ is independent of $\gamma$. The estimate holds for all $\vec{c}(t) \in B_{R_{0}, \gamma}$. Proceed with the estimate for the operator $R$. In view of (32) we have that

$$
\begin{gathered}
\left\|R\left(c^{1}\right)-R\left(c^{2}\right)\right\|_{L_{p}(0, \gamma)} \leq \\
\leq c \sum_{j=1}^{r}\left\|L^{-1} M_{r_{0}}\left(V^{1}-V^{2}\right)\left(x_{j}, t\right)+\sum_{i=r_{0}+1}^{r} c_{i}^{1} L^{-1} M_{i} V^{1}\left(x_{j}, t\right)-c_{i}^{2} L^{-1} M_{i} V^{2}\left(x_{j}, t\right)\right\|_{L_{p}(0, \gamma)} \leq \\
\leq \sum_{j=1}^{r}\left(\left\|\sum_{i=r_{0}+1}^{r}\left(c_{i}^{1}-c_{i}^{2}\right)(t) L^{-1} M_{i} V^{1}\left(x_{j}, t\right)\right\|_{L_{p}(0, \gamma)}+\right. \\
\left.+\left\|c_{i}^{2}\left(L^{-1} M_{i} V^{1}\left(x_{j}, t\right)-L^{-1} M_{i} V^{2}\left(x_{j}, t\right)\right)\right\|_{L_{p}(0, \gamma)}\right)+c_{4}\left\|\overrightarrow{c^{1}}-\overrightarrow{c^{2}}\right\|_{L_{p}(0, \gamma)} .
\end{gathered}
$$

We estimate the first summands on the right-hand side as follows:

$$
\left\|\left(c_{i}^{1}-c_{i}^{2}\right) L^{-1} M_{i} V^{1}\left(x_{j}, t\right)\right\|_{L_{p}(0, \gamma)} \leq\left\|c_{i}^{1}-c_{i}^{2}\right\|_{L_{p}(0, \gamma)}\left\|L^{-1} M_{i} V^{1}\left(x_{j}, t\right)\right\|_{L_{\infty}(0, \gamma)} .
$$

By the embedding theorems and Theorem 1, we derive that

$$
\begin{gathered}
\left\|L^{-1} M_{i} V^{1}\left(x_{j}, t\right)\right\|_{L_{\infty}(0, \gamma)} \leq\left\|M_{i} V^{1}\right\|_{L_{\infty}\left(0, \gamma ; L_{p}(G)\right)} \leq \\
c\left\|V^{1}(x, t)\right\|_{L_{\infty}\left(0, \gamma ; W_{p}^{2}(G)\right)} \leq c \gamma^{1 / q}\left\|V_{t}^{1}\right\|_{L_{p}\left(0, \gamma ; W_{p}^{2}(G)\right)}\left(\frac{1}{q}+\frac{1}{p}=1\right) .
\end{gathered}
$$

Next, we have the estimate

$$
\left\|\left(c_{i}^{1}-c_{i}^{2}\right) L^{-1} M_{i} V^{1}\left(x_{j}, t\right)\right\|_{L_{q}(0, \gamma)} \leq c_{1} \gamma^{1 / q}\left\|\overrightarrow{c^{1}}-\overrightarrow{c^{2}}\right\|_{L_{q}(0, \gamma)},
$$

where constant $c_{1}$ depends on $R_{0}$, but independent of $\gamma$. Similarly, we justify the inequality

$$
\begin{gathered}
\left\|c_{i}^{2}\left(L^{-1} M_{i}\left(V^{1}\left(x_{j}, t\right)-V^{2}\left(x_{j}, t\right)\right)\right)\right\|_{L_{q}(0, \gamma)} \leq R_{0}\left\|V^{1}(x, t)-V^{2}(x, t)\right\|_{L_{\infty}\left(0, \gamma ; W_{p}^{2}(G)\right)} \leq \\
\leq R_{0} c \gamma^{1 / q}\left\|V_{t}^{1}-V_{t}^{2}\right\|_{L_{p}\left(0, \gamma ; W_{p}^{2}(G)\right)} \leq c_{1} \gamma^{1 / q}\left\|\overrightarrow{c^{1}}-\overrightarrow{c^{2}}\right\|_{L_{p}(0, \gamma)} .
\end{gathered}
$$

From (34), (35), and (33) it follows that

$$
\left\|R\left(c^{1}\right)-R\left(c^{2}\right)\right\|_{L_{p}(0, \gamma)} \leq c \gamma^{1 / q}\left\|\overrightarrow{c^{1}}-\overrightarrow{c^{2}}\right\|_{L_{p}(0, \gamma)} .
$$

Choose $\gamma_{0}$ so that

$$
c \gamma_{0}^{1 / q}=1 / 2
$$

In this case, for all

$$
c^{1}, c^{2} \in B_{R_{0}, \gamma}
$$


with $\gamma \leq \gamma_{0}$, we have inequality (36). Moreover,

$$
R(\vec{c})=R(\vec{c})-R(0)+R(0)
$$

and

$$
\|R(\vec{c})\|_{L_{q}(0, \gamma)} \leq\|R(0)\|_{L_{q}(0, \gamma)}+\frac{1}{2}\|\vec{c}\|_{L_{q}(0, \gamma)} \leq \frac{R_{0}}{2}+\frac{R_{0}}{2}=R_{0} .
$$

Thus, $R$ takes the ball $B_{R_{0}, \gamma_{0}}$ into itself and is contractive. By the fixed-point theorem, equation (26) is solvable. By construction, $V$ is a solution to (18). The fact that $V$ meets (19) is proven by analogy with arguments those of in the linear case (see [30]).

Conclusion. Thus, we proved that the inverse problems under consideration are well-posed at least locally in time. The results obtained allow to construct new numerical algorithms for solving problems of form (1) - (4).

The authors were supported by the Russian Foundation for Basic Research (Grant 15-0106582).

\section{References / Литература}

1. Lyubanova A.Sh., Tani A. On Inverse Problems for Pseudoparabolic and Parabolic Equations of Filtration. Inverse Problems in Science and Engineering, 2011, vol. 19, no. 7, p. 1023-1042. DOI: $10.1080 / 17415977.2011 .569712$

2. Sveshnikov A.G., Alshin A.B., Korpusov M.O., Pletner U.D. Linear and Non-Linear SobolevType Equations. FML, 2007.

3. Bebernes J., Lacey A.A. Global Existence and Finite Time Blow-Up for a Class of Nonlocal Parabolic Problems. Adv. Differ. Equations, 1997, vol. 2, pp. 927-954.

4. Sviridyuk G.A., Fedorov V.E. Linear Sobolev Type Equations and Degenerate Semigroups of Operator. Utrecht: VSP, 2003. 228 p. DOI: 10.1515/9783110915501

5. Chung S.K., Pani A.K. Numerical Methods for the Rosenau Equation. L. Appl. Anal., 2001, vol. 77, no. 3-4, pp. 100-116. DOI: 10.1080/00036810108840914

6. Chen Yu. Remark on the Global Existence for the Generalized Benjamin-Bona-Mahony Equations in Arbitrary Dimension. Appl. Anal., 1988, vol. 30, no. 1-3, pp. 1-15. DOI: $10.1080 / 00036818808839789$

7. Egorov I.E., Pyatkov S.G., Popov S.V. Nonclassical Operator-Differential Equations. Novosibirsk, Nauka, 2000. 336 р. [Егоров, И.Е. Неклассические дифференциальнооператорные уравнения / И.Е. Егоров, С.Г. Пятков, С.В. Попов. - Новосибирск: Наука, 2000. -336 c.]

8. Gajewski H. Groger K. Zacharias K. Nichtlineare Operatorgleichungen und Operatordifferentialgleichungen. Berlin, Akademie-Verlag, 1974.

9. Showalter R.E. Monotone Operators in Banach Space and Nonlinear Partial Differentail Equations. Providence, AMS, 1997. 278 p.

10. Kozhanov A.I. An Initial-boundary Value Problem for Equations of the Generalized Boussinesq Equation Type with a Nonlinear Source. Math. Notes, 1999, vol. 65, no. 1, pp. 59-63. DOI: $10.1007 / \mathrm{BF} 02675010$

11. Gladkov A.L. Unique Solvability of the Cauchy Problem for Certain Quasilinear Pseudoparabolic Equations. Math. Notes, 1996, vol. 60, no. 3, pp. 264-268. DOI: $10.1007 / \mathrm{BF} 02320362$ 
12. Di Benedetto E., Pierre M. On the Maximum Principle for Pseudoparabolic Equations. Indiana Univ. Math. J., 1981, vol. 30, no. 6, pp. 821-854. DOI: 10.1512/iumj.1981.30.30062

13. Begehr H., Dai D. Q. Initial Boundary Value Problem for Nonlinear Pseudoparabolic Equations. Complex Variables, Theory Appl., 1992, vol. 18, no. 1-2, pp. 33-47.

14. Mitidieri E., Pohozhaev S.I. A Priori Estimates and the Absence of Solutions of Nonlinear Equations and Inequalities of Partial. Proceedings of the Steklov Institute of Mathematics, 2001, vol. 234, no. 3, pp. 1-362.

15. Laptev G.G. About the Absence of Solutions for One Class of Singular Semilinear Differential Inequalities. Proceedings of the Steklov Institute of Mathematics, 2001, vol. 232, pp. 216-228.

16. Lyubanova A.Sh. Identification of a Coefficient in the Leading Term of a Pseudoparabolic Equation of Filtration. Siberian Mathematical Journal, 2013, vol. 54, no. 6, pp. 1048-1058. DOI: $10.1134 /$ S0037446613060116

17. Asanov A., Atamanov E.R. An Inverse Problem for a Pseudoparabolic Integro-defferential Operator Equation. Siberian Mathematical Journal, 1995, vol. 38, no. 4, pp. 645-655. DOI: $10.1007 / \mathrm{BF} 02107322$

18. Mamayusupov M.Sh. The Problem of Determining Coefficients of a Pseudoparabolic Equation. Studies in Integro-differential Equations, Ilim, Frunze, 1983, no. 16, pp. 290-297.

19. Favini A., Lorenzi A. Differential Equations. Inverse an Direct Problems. Tylor \& Francis Group, LLC. 2006. DOI: 10.1201/9781420011135

20. Urazaeva A.V., Fedorov V.E. On the Well-Posedness of the Prediction-Control Problem for Certain Systems of Equations. Math. Notes, 2009, vol. 85, no. 3, pp. 426-436. DOI: $10.1134 /$ S0001434609030134

21. Kozhanov A.I. Composite Type Equations and Inverse Problems. Utrecht, VSP, 1999. DOI: $10.1515 / 9783110943276$

22. Kabanikhin S.I. Inverse and Incorrect Problem. Novosibirsk: Siberian science publishing, 2009. 457 p. (in Russian) [Кабанихин С.И. Обратные и некоректные задачи. Новосибирск: Сибирское научное издательство, 2009. - 457 с.]

23. Belov Ya.Ya. Inverse Problems for Parabolic Equations. Utrecht, VSP, 2002.

24. Ivanchov M. Inverse Problems for Equations of Parabolic Type. Math. Studies. Monograph Series. V. 10. Lviv, WNTL Publishers, 2003.

25. Isakov V. Inverse Problems for Partial Differential Equations. Berlin, Springer, 2006.

26. Prilepko A.I., Orlovsky D.G., and Vasin I.A. Methods for Solving Inverse Problems in Mathematical Physics. N.Y., Marcel Dekker, Inc. 1999.

27. Pyatkov S.G. On Some Classes of Inverse Problems for Parabolic Equations. J. Inv. Ill-Posed Problems, 2011, vol. 18, no. 8, pp. 917-934.

28. Pyatkov S.G., Samkov M.L. On Some Classes of Coefficient Inverse Problems for Parabolic Systems of Equations. Sib. Adv. in Math., 2012, vol. 22, no. 4, pp. 287-302. DOI: $10.3103 / \mathrm{S} 1055134412040050$

29. Pyatkov S.G., Tsybikov B.N. On Some Classes of Inverse Problems for Parabolic and Elliptic Equations. J. Evol. Equat., 2011, vol. 11, no. 1, pp. 155-186. DOI: 10.1007/s00028-010-0087-6

30. Shergin S.N., Pyatkov S.G. On Some Classes of Inverse Problems for Pseudoparabolic Equations. Matem. Zam. SVFU., 2014. vol. 21, no. 2, pp. 106-116. (in Russian) [Шергин, С.Н. О некоторых классах обратных задач для псевдопараболических уравнений / С.Н. Шергин, С.Г. Пятков // Математические заметки СВФУ. - 2014. - Т. 21, №2. C. $106-116$. 
31. Gilbarg D., Trudinger N. Elliptic Differential Equation with Partial Derivative of the Second Order. Berlin, Heidelberg, Springer-Verlag, 2001.

32. Triebel H. Interpolation Theory. Function Space. Differential Operator. Berlin, VEB Deutscher Verlag der Wissenschaften, 1978. 528 p.

33. Krylov N.V. Lectures on Elliptic and Parabolic Equations in Sobolev Spaces. AMS, 2008. DOI: $10.1090 / \mathrm{gsm} / 096$

34. Ladyzhenskaya O.A, Ural'tseva N.N. Linear and Quasilinear Elliptic Equations. Moscow: Nauka, 1964. [Ладыженская, О.А. Линейные и квазилинейные уравнения эллиптического типа / О.А. Ладыженская, Н.Н. Уральцева. - М.: Наука, 1973.]

35. Amann H. Operator-Valued Foutier Multipliers, Vector-valued Besov Spaces and Applications. Mathem. Nachr., 1997, vol. 186, no. 1, pp. 5-56.

DOI: $10.1002 /$ mana.3211860102

Received December 27, 2014

УДК 517.95

DOI: $10.14529 / \mathrm{mmp} 150209$

\title{
НЕКОТОРЫЕ МАТЕМАТИЧЕСКИЕ МОДЕЛИ ФИЛЬТРАЦИОННОЙ ТЕОРИИ
}

\author{
С.Г. Пятков, С.Н. Шергин
}

Работа посвящена рассмотрению обратных задач для некоторых математических моделей, возникающих в теории фильтрации. Мы рассматриваем обратную задачу об определении неизвестной правой части и коэффициентов в псевдопараболическом уравнении третьего порядка. Уравнения такого типа и более общие уравнения Соболевского типа возникают в теории фильтрации, при описании процессов тепло и массопереноса, физике плазмы и во многих других областях. Задача сводится к некоторому операторному уравнению, разрешимость которого устанавливается при помощи априорных оценок и теоремы о неподвижной точке. Кроме естественных условий гладкости данных, мы требуем также выполнения некоторого условия корректности, которое по существу сводится к условию невырожденности некоторой матрицы, построенной по данным задачи. Формулируются и доказываются теоремы о существовании и единственности решения поставленной задачи. Приводится оценка устойчивости. В линейном случае результат является глобальным по времени, а в нелинейном локальным по времени. В качестве основных пространств рассматриваются пространства С.Л. Соболева.

Ключевые слова: уравнение псевдопараболического типа; теорема существования и единственности решения; обратная задача; краевая задача.

Сергей Григорьевич Пятков, доктор физико-математических наук, профессор, кафедра высшей математики, Югорский государственный университет (г. Ханты-Мансийск, Российская Федерация), S_pyatkov@ugrasu.ru.

Сергей Николаевич Шергин, аспирант, кафедра высшей математики, Югорский государственный университет (г. Ханты-Мансийск, Российская Федерация), ssn@ugrasu.ru.

Поступила в редакиию 27 декабря 2014 г. 